\title{
GW4064 attenuates lipopolysaccharide-induced hepatic inflammation and apoptosis through inhibition of the Toll-like receptor 4-mediated p38 mitogen-activated protein kinase signaling pathway in mice
}

\author{
HSUAN-MIAO LIU ${ }^{1}$, TZUNG-YAN LEE ${ }^{2-4}$ and JYH-FEI LIAO ${ }^{1}$ \\ ${ }^{1}$ Department and Institute of Pharmacology, National Yang-Ming University, 112 Taipei; \\ ${ }^{2}$ Graduate Institute of Traditional Chinese Medicine, School of Chinese Medicine, College of Medicine, \\ Chang Gung University, 333 Taoyuan; ${ }^{3}$ Department of Traditional Chinese Medicine, \\ Chang Gung Memorial Hospital, 204 Keelung; ${ }^{4}$ Department of Medical Research, \\ China Medical University Hospital, China Medical University, 404 Taichung, Taiwan, R.O.C.
}

Received June 16, 2017; Accepted January 4, 2018

DOI: $10.3892 /$ ijmm.2018.3366

\begin{abstract}
Liver injury is associated with devastating consequences caused by inflammation and apoptosis. The farnesoid $\mathrm{X}$ receptor (FXR) is a nuclear receptor that has an essential role in hepatoprotection by maintaining the homeostasis of liver metabolism. The present study investigated the capacity of the FXR agonist GW4064 to protect the livers of mice from lipopolysaccharide (LPS)-induced inflammation and apoptosis. Male C57BL/6J [wild-type (WT)] and FXR knockout (KO) mice were intraperitoneally injected with LPS or saline. LPS-treated mice were intraperitoneally injected with vehicle or GW4064 $(20 \mathrm{mg} / \mathrm{kg})$ twice and then sacrificed. Activation of FXR by GW4064 alleviated hepatic inflammation in the LPS-induced murine liver injury model as reflected by reduced serum levels of aspartate aminotransferase and pro-inflammatory cytokine mRNA expression, including tumor necrosis factor- $\alpha$, as well as interleukin- 6 and $-1 \beta$ in WT mice. In addition, Toll-like receptor 4 (TLR4), p38 mitogen-activated protein kinase (MAPK), B-cell lymphoma-2-associated $\mathrm{X}$ protein and cytochrome $c$ protein levels were decreased in WT mice receiving LPS with simultaneous GW4064 administration compared with those receiving LPS alone, while this
\end{abstract}

Correspondence to: Professor Tzung-Yan Lee, Graduate Institute of Traditional Chinese Medicine, School of Chinese Medicine, College of Medicine, Chang Gung University, 259 Wen-Hwa 1st Road, Kwei-Shan, 333 Taoyuan, Taiwan, R.O.C.

E-mail: joyamen@mail.cgu.edu.tw

Professor Jyh-Fei Liao, Department and Institute of Pharmacology, National Yang-Ming University, 155 Linong Street, Taipei, 112 Taiwan, Taiwan, R.O.C.

E-mail: jfliao@ym.edu.tw

Key words: farnesoid X receptor, GW4064, lipopolysaccharide, Toll-like receptor 4 , inflammation was not observed in FXR KO mice. These results indicated that in WT mice, administration of GW4064 ameliorated LPS-mediated liver injury by upregulation of FXR expression, which was in part mediated by the TLR4/p38 MAPK pathway.

\section{Introduction}

Dysregulation of innate immunity and liver inflammation are triggered by the translocation of gut-derived endotoxins, such as lipopolysaccharides (LPS), to the liver (1). LPS-induced experimental animal models are a common means of studying liver injury, fibrosis and sepsis (2). Liver injury triggered by LPS has been identified to be associated with certain biological processes, including inflammation and apoptosis via specific signaling pathways. Toll-like receptor 4 (TLR4), one receptor for LPS, may trigger the myeloid differentiation factor 88 (MyD88)-dependent pathway, which leads to the activation of nuclear factor (NF)- $\mathrm{B}$ and activates the mitogen-activated protein kinase (MAPK) pathways (p38, extracellular signal-regulated kinase and c-Jun N-terminal kinase) (3), which stimulates the production of proinflammatory cytokines, chemokines and type I interferon (4). It has been reported that suppression of TLR4 and MyD88 inhibits the translocation of $\mathrm{NF}-\kappa \mathrm{B}$ and affects the levels of interleukin-1 $\beta$ (IL-1 $\beta$ ), IL-6 and tumor necrosis factor $\alpha(\mathrm{TNF}-\alpha)(5)$. These inflammatory mediators contribute to hepatocyte dysfunction, apoptosis and necrosis, and the generation of extracellular matrix proteins, leading to characteristic fibrosis (6).

Farnesoid X receptor (FXR) has a key role in regulating fatty acid and glucose metabolism and also has an important role in maintaining cholesterol and bile acid levels $(7,8)$. In addition, FXR has been demonstrated to have anti-inflammatory functions, and agonist-activated FXR was reported to inhibit NF- $\kappa \mathrm{B}$ target inflammatory genes in hepatocytes (9). A previous study reported that FXR knockout (KO) mice displayed prominent liver injury and inflammation, and developed spontaneous liver tumors as they aged (10). A similar study indicated that 
the expression of inflammatory genes in the liver was elevated in FXR KO mice (11). GW4064, a synthetic FXR ligand, has been reported to alleviate LPS-induced hepatic inflammation by repressing macrophage activation (12) and protects liver cells from apoptosis induced by serum deprivation in vitro and fasting in vivo (13). In addition, activation of FXR was reported to have hepatoprotective effects against certain toxins, which may be a key function of FXR(12).

The present study demonstrated that administration of FXR agonist GW4064 in mice reduced the severity of LPS-induced liver injury, probably through its anti-inflammatory properties. The present study revealed novel role for FXR in the control of liver inflammation by antagonizing the TLR4 signaling pathway. Based on the results, FXR was suggested to be a potential regulator of hepatic inflammation and apoptosis and GW4064 may be used to treat liver inflammatory diseases. This hepatoprotection by GW4064 was totally abolished in FXR KO mice. The unanticipated link between FXR and the TLR4/p38 MAPK/NF- $\mathrm{B}$ axis highlights a novel mechanism that contributes to the hepatoprotective effects of FXR activators.

\section{Materials and methods}

Animals and experimental design. A total of 15 male C57BL/6J wild-type (WT) mice (weight, 24-28 g; age, 13 weeks) were obtained from the National Laboratory Animal Center (Taiwan) and 15 FXR-knockout (KO) mice (B6.129X1(FVB)-Nrlh4 $4^{\text {tmlGonz }} / \mathrm{J}$; stock no. 007214; weight, 24-28 g; age, 13 weeks) were obtained from the Jackson Laboratory. Mice were assigned to 6 groups ( $n=5$ per group): i) C57BL/6J wild-type mice; ii) LPS-treated wild-type mice; iii) LPS-treated wild-type mice intraperitoneally injected with GW4064; iv) FXR KO mice; v) LPS-treated FXR KO mice; vi) LPS-treated FXR KO mice were intraperitoneally injected with GW4064. All animals were housed under a 12-h light-dark cycle and were provided a standard laboratory diet ad libitum. The study protocol was reviewed and approved by the Animal Care and Use Committee of Chang Gung University (Taoyuan, Taiwan) and was in accordance with the guidelines of the NIH Guide for the Care and Use of Laboratory Animals. LPS (cat. no. L2880; Sigma-Aldrich; Merck KGaA, Darmstadt, Germany) was administered intraperitoneally (i.p.) at $5 \mathrm{mg} / \mathrm{kg}$, and was applied to induce inflammation-associated disease as previously described $(14,15)$. WT and FXR KO mice were intraperitoneally injected with a single dose of LPS $(5 \mathrm{mg} / \mathrm{kg})$ and were intraperitoneally injected with GW4064 twice $24 \mathrm{~h}$ later (20 mg/kg i.p.; cat. no. G5172; Sigma-Aldrich, Merck $\mathrm{KGaA})(16,17)$. In a Fisher rat model, GW4064 was reported to lower serum triglyceride levels in a dose-dependent manner and to have a median effective dose of $20 \mathrm{mg} / \mathrm{kg}$ (18). All mice were euthanized $6 \mathrm{~h}$ after the last GW4064 administration with carbon dioxide. Blood samples were collected for determining serum alanine transaminase (ALT), and liver tissues were exsanguinated, immediately frozen in liquid nitrogen and stored at $-80^{\circ} \mathrm{C}$ for further analysis.

Serum ALT. Serum levels of ALT were determined by spectrophotometric analysis utilizing the Randox ALT assay kit (cat. no. AL1205; Randox, Antrim, UK) according to the manufacturer's instructions.
Histology and immunohistochemistry. For morphological analysis, murine liver specimens were fixed in $4 \%$ paraformaldehyde, embedded in paraffin and cut into 5- $\mu \mathrm{m}$ sections. Slides were deparaffinized according to standard procedures and stained with freshly made hematoxylin (cat. no. H3136) and eosin (H\&E; cat. no. 230251) (both from Sigma-Aldrich; Merck KGaA) followed by microscopic examination. For $\mathrm{H} \& \mathrm{E}$ staining, tissues were counterstained for $10 \mathrm{~min}$ at room temperature $\left(20-30^{\circ} \mathrm{C}\right)$. For protein detection, sections were rehydrated with PBS prior to being incubated in $3 \%$ $\mathrm{H}_{2} \mathrm{O}_{2}$ /PBS (cat. no. 31642; Sigma-Aldrich; Merck KGaA;) to block endogenous peroxidase and blocked in 5\% normal goat serum (cat. no. 566380; Sigma-Aldrich; Merck KGaA) in PBS, followed by staining with primary antibodies overnight at $4^{\circ} \mathrm{C}$. The following primary antibodies were used: Anti-FXR (cat. no. SC-13063; 1:90; Santa Cruz Biotechnology, Inc., Dallas, TX, USA), TLR4 (cat. no. ab47093; 1:100; Abcam, Cambridge, UK), MyD88 (cat. no. AB16529; 1:90; EMD Millipore, Billerica, MA, USA), phospho-p38 MAPK (pT18/pY182; cat. no. 612280; 1:100; BD Biosciences, Santa Clara, CA, USA), NF-кB (cat. no. SC-8008; 1:90; Santa Cruz Biotechnology, Inc. Dallas, TX, USA), Bax (cat. no. ab7977; 1:100) and cytochrome $c$ (cat. no. ab13575; 1:100) (both from Abcam). Anti-mouse (cat. no. AP124P; 1:100; Sigma-Aldrich; Merck KGaA) and rabbit (cat. no. ab6721; 1:100; Abcam) horseradish peroxidase (HRP)-conjugated secondary antibodies were used for $1 \mathrm{~h}$ at room temperature $\left(20-30^{\circ} \mathrm{C}\right)$. Finally, immunostaining was visualized by application of a 3,3'-diaminobenzidine (CN/DAB) substrate kit (cat. no. 34000; Thermo Fisher Scientific, Inc., Waltham, MA, USA) and viewed under a light microscope (XI71; Olympus, Tokyo, Japan).

Protein isolation and western blot analysis. Mouse liver tissues were lysed in lysis buffer [20 mM Tris (pH 7.4), $150 \mathrm{mM} \mathrm{NaCl}$, $1 \%$ Triton X-100, 1 mM EDTA, 1 mM EGTA, $2.5 \mathrm{mM}$ sodium pyrophosphate, $1 \mathrm{mM} \beta$-glycerophosphate, $1 \mathrm{mM}$ sodium orthovanadate] containing protease inhibitor cocktail (cat. no. 78429; Thermo Fisher Scientific, Inc.). The Thermo Scientific NE-PER Nuclear and Cytoplasmic Extraction kit (cat. no. 78835; Thermo Fisher Scientific, Inc.) provides for efficient cell lysis and extraction of separate cytoplasmic and nuclear protein fractions. Lysates were clarified by centrifugation at $10,000 \mathrm{x}$ for $10 \mathrm{~min}$ at $4^{\circ} \mathrm{C}$ and the protein concentration was determined using a Bio-Rad Protein assay (cat. no. 500-0006; Bio-Rad Laboratories, Inc., Hercules, CA, USA). Protein was analyzed by western blot analysis. The boiled samples were separated by $10 \%$ SDS-PAGE and transferred onto nitrocellulose membranes (cat. no. 88018; Thermo Fisher Scientific, Inc.). After blocking with 5\% non-fat skimmed milk or bovine serum albumin (cat. no. A1933; Sigma-Aldrich; Merck $\mathrm{KGaA}$ ) in Tris-buffered saline with $0.1 \%$ Tween-20 for $1 \mathrm{~h}$, the membranes were probed with the corresponding antibodies overnight at $4^{\circ} \mathrm{C}$. The following primary antibodies were used for western blotting: Anti-FXR (cat. no. SC-13063; 1:500; Santa Cruz Biotechnology, Inc.); TLR4 (cat. no. ab47093; 1:1,000; Abcam); NF-кB (cat. no. SC-8008; 1:1,000); B-cell lymphoma-2 (Bcl-2; cat. no. SC-7382; 1:750) (both from Santa Cruz Biotechnology, Inc.) and Bcl-2-associated X protein (Bax; cat. no. ab7977; 1:1,000; Abcam). $\beta$-actin (cat. no. MA5-15739; 1:1,000; Thermo Fisher Scientific, 
Table I. Sequences of primers used for reverse transcriptionquantitative polymerase chain reaction.

\begin{tabular}{lcc}
\hline Gene & Direction & Primer sequence \\
\hline TNF- $\alpha$ & F & 5'-TTGACCTCAGCGCTGAGTTG-3' \\
& R & 5'-CCTGTAGCCCACGTCGTAGC-3' \\
IL-1 $\beta$ & F & 5'-GCAACTGTTCCTGAACTCA-3' \\
& R & 5'-CTCGGAGCCTGTAGTGCAG-3' \\
IL-6 & F & 5'-GTACTCCAGAAGACCAGAGG-3' \\
& R & 5'-TGCTGGTGACAACCACGGCC-3' \\
IFN- $\gamma$ & F & 5'-AGCAACAACATAGCGTCAT-3' \\
& R & 5'-CCTCAAACTTGGCAATACTC-3' \\
FXR & F & 5'-GGCCTCTGGGTACCACTACA-3' \\
& R & 5'-AAGAAACATGGCCTCCACTG-3' \\
SHP & F & 5'-ACTGGCTGCAGTTCAGTGGC-3' \\
& R & 5'-GGTGAAGAGGATCGTGCCC-3' \\
BAX & F & 5'-TTTGCTTCAGGGTTTCATCC-3' \\
& R & 5'-CAGTTGAAGTTGCCGTCAGA-3' \\
BCL-2 & F & 5'-TCTTTGAGTTCGGTGGGGTC-3' \\
& R & 5'-TGCATATTTGTTGGGGCAGG-3' \\
Bcl-xL & F & 5'-TTGGACAATGGACTGGTTGA-3' \\
GAPDH & R & 5'-GTAGAGTGGATGGTCAGTG-3' \\
& R & 5'-TCACCACCATGGAGAAGGC-3' \\
& 5'-GCTAAGCAGTTGGTGGGCA-3'
\end{tabular}

F, forward; R, reverse; TNF- $\alpha$, tumor necrosis factor- $\alpha$; IL- $1 \beta$, interleukin-1 $\beta$; IFN- $\gamma$, interferon- $\gamma$; FXR, farnesoid $\mathrm{X}$ receptor; SHP, small heterodimer partner; Bcl-2, B-cell lymphoma 2; Bax, Bcl-2-associated X protein; Bcl-xL, Bcl extra-large protein; GAPDH, glyceraldehyde 3-phosphate dehydrogenase.

Inc.) and histone H1 (cat. no. SC-56695; 1:1,000; Santa Cruz Biotechnology, Inc.) antibodies were used to confirm equal protein loading for all samples. Anti-mouse (cat. no. AP124P; 1:5,000; EMD Millipore) and rabbit (cat. no. ab6721; 1:5,000; Abcam) horseradish peroxidase (HRP)-conjugated secondary antibodies were used for $1 \mathrm{~h}$ at room temperature. Bound HRP-conjugated antibodies were visualized by enhanced chemiluminescence (cat. no. 34080; Thermo Fisher Scientific, Inc.). Quantification of the blots was performed using ImageQuant 5.2 software (GE Healthcare, Little Chalfont, UK) and calibrated using $\beta$-actin or histone as an internal control.

Reverse transcription-quantitative polymerase chain reaction $(R T-q P C R)$. Total RNA was extracted from the liver tissue using TRIzol reagent (cat. no. 15596018; Thermo Fisher Scientific, Inc.) according to the manufacturer's instructions, and the concentration and integrity of the isolated RNA were determined at the optical density at 260/280 nm. Complementary (c)DNA was synthesized from total RNA (4 $\mu \mathrm{g})$ using the RevertAid ${ }^{\mathrm{TM}}$ First Strand cDNA Synthesis kit (cat. no. 00187457; Thermo Fisher Scientific, Inc.), followed by qPCR using the LightCycler ${ }^{\circledR} 480$ SYBR-Green I Master (cat. no. 04707516001; Roche Applied Science, Penzberg, Germany) on a LightCycler 1.5 apparatus (cat. no. 03515885001; Roche
Applied Science, Penzberg, Germany). PCR was performed using the following conditions: $95^{\circ} \mathrm{C}$ for $10 \mathrm{~min}$, followed by 45 cycles of $95^{\circ} \mathrm{C}$ for $15 \mathrm{sec}, 57^{\circ} \mathrm{C}$ for $30 \mathrm{sec}$ and $72^{\circ} \mathrm{C}$ for $30 \mathrm{sec}$. The qPCR data were normalized to GAPDH expression as an internal control. The sequences of primers used for RT-qPCR are listed in Table I.

Statistical analysis. Values are expressed as the mean \pm standard error of the mean. The statistical analyses were performed using one-way analysis of variance followed by the Student Newman-Keuls multiple-range test. SAS v9.4 software was used for statistical analysis (SAS Institute, Inc., Cary, NC, USA). $\mathrm{P}<0.05$ was considered to indicate a statistically significant difference.

\section{Results}

Effects of GW4064 on serum ALT, histopathological changes and inflammatory responses in the liver. To evaluate whether activation of FXR had a protective effect on LPS-induced liver injury, WT and FXR KO mice were used and a highly potent FXR agonist, GW4064, was applied in the present study. H\&E staining revealed that the liver parenchymal cells were homogeneously swollen and that hypertrophy and multiple focal hepatic necrosis were present in LPS-treated mice. FXR KO mice had enlarged livers, whereas those injected with LPS developed more severe liver injury and hepatocyte ballooning (Fig. 1A). FXR KO mice exhibited increased serum ALT levels compared with WT mice (Fig. 1B), while GW4064 administration in LPS-injected animals resulted in apparently normal hepatocytes in liver sections (Fig. 1A), as evidenced by a significant decline in the increase of serum ALT levels in WT mice, whereas no beneficial effect was seen in FXR KO mice (Fig. 1B). To further address whether FXR may modulate inflammatory gene expression in mice, the present study compared the induction of pro-inflammatory cytokines by LPS in WT and FXR KO mice. Of note, GW4064 attenuated liver injury as indicated by inhibition of LPS-induced increases in pro-inflammatory cytokines in WT mice, but not in FXR KO mice (Fig. 1C).

Effects of GW4064 on FXR and small heterodimer partner (SHP) levels in liver tissue. Next, the present study determined whether FXR expression was affected upon LPS injection in mice. LPS injection reduced FXR protein levels and mRNA expression in WT mice (Fig. 2). Compared with WT mice, FXR KO mice displayed lowed FXR and SHP mRNA expression in the liver. In addition, GW4064 significantly inhibited the LPS-mediated reduction in FXR protein levels and SHP mRNA expression in WT mice, whereas no effect was observed in FXR KO mice after LPS or GW4064 administration (Fig. 2).

GW4064 represses LPS-induced hepatic TLR4/MyD88/p38 $M A P K / N F-\kappa B$ signaling in mice. The present study further determined the role of FXR in the LPS-induced TLR4/MyD88 pathway in the livers of WT and FXR KO mice. GW4064 significantly repressed LPS-induced TLR4 (Fig. 3A) and MyD88 (Fig. 3B) protein levels in WT mice. TLR4 (Fig. 3A) and MyD88 (Fig. 3B) protein levels were higher in FXR KO mouse livers 
A

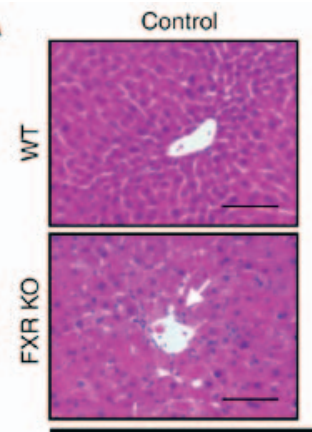

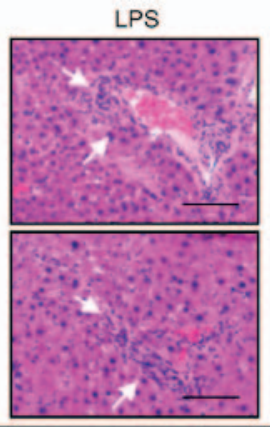

$\mathrm{H} \& \mathrm{E}$

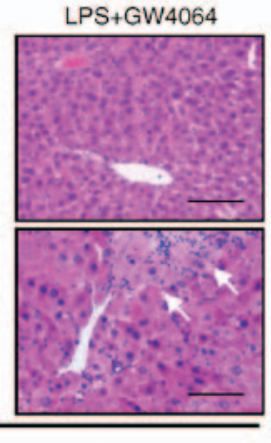

IL-1 $1 \beta$
IL-6
B

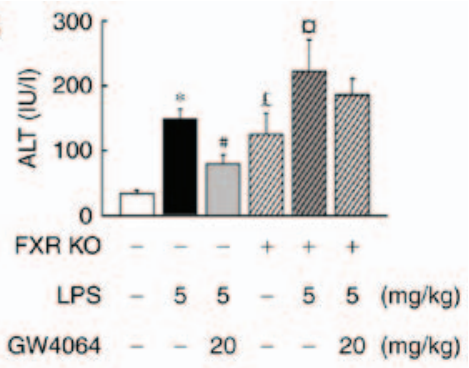

C

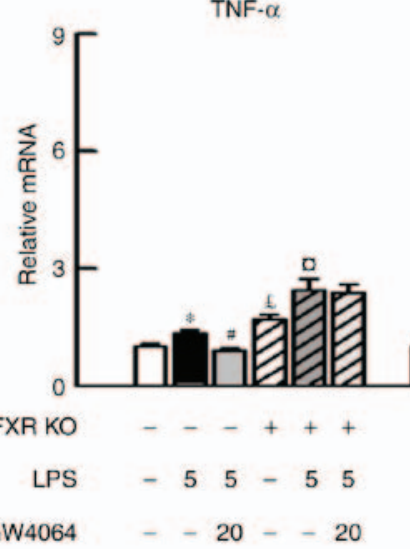

$-20--20$

$-+5-55$

$-55-55(\mathrm{mg} / \mathrm{kg})$

GW4064

Figure 1. GW4064 ameliorates LPS-induced liver injury and hepatic inflammation in WT mice. WT and FXR KO mice were treated with or without GW4064 $(20 \mathrm{mg} / \mathrm{kg})$ twice after LPS injection $(5 \mathrm{mg} / \mathrm{kg}$ ). All mice were sacrificed after the last GW4064 injection. (A) Fresh WT mouse liver tissues were fixed in $4 \%$ paraformaldehyde and $5-\mu \mathrm{m}$ sections were stained with H\&E. Hepatocyte inflammation and damage was histopathologically analyzed (indicated by white arrows; magnification, x200; scale bar, $100 \mu \mathrm{m}$ ). (B) Serum ALT levels in the livers of WT and FXR KO mice were determined. (C) Total RNAs from liver tissues were isolated and hepatic mRNA expression of inflammatory cytokines was determined by reverse transcription-quantitative polymerase chain reaction analysis. Values are expressed as the mean \pm standard error of the mean ( $\mathrm{n}=5$ mice for each group). " $\mathrm{P}<0.05$, WT vs. LPS; ${ }^{~} \mathrm{P}<0.05$, LPS vs. LPS+GW4064; ${ }^{\mathfrak{f}} \mathrm{P}<0.05$, WT vs. FXR KO; ${ }^{\circ} \mathrm{P}<0.05$, FXR KO vs. FXR KO+LPS. WT, wild-type; KO, knockout; FXR, farnesoid X receptor; LPS, lipopolysaccharide; H\&E, hematoxylin and eosin; ALT, alanine transaminase; TNF, tumor necrosis factor; IL, interleukin; IFN, interferon.

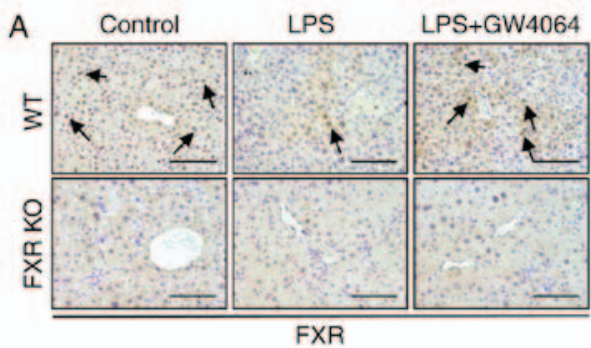

B
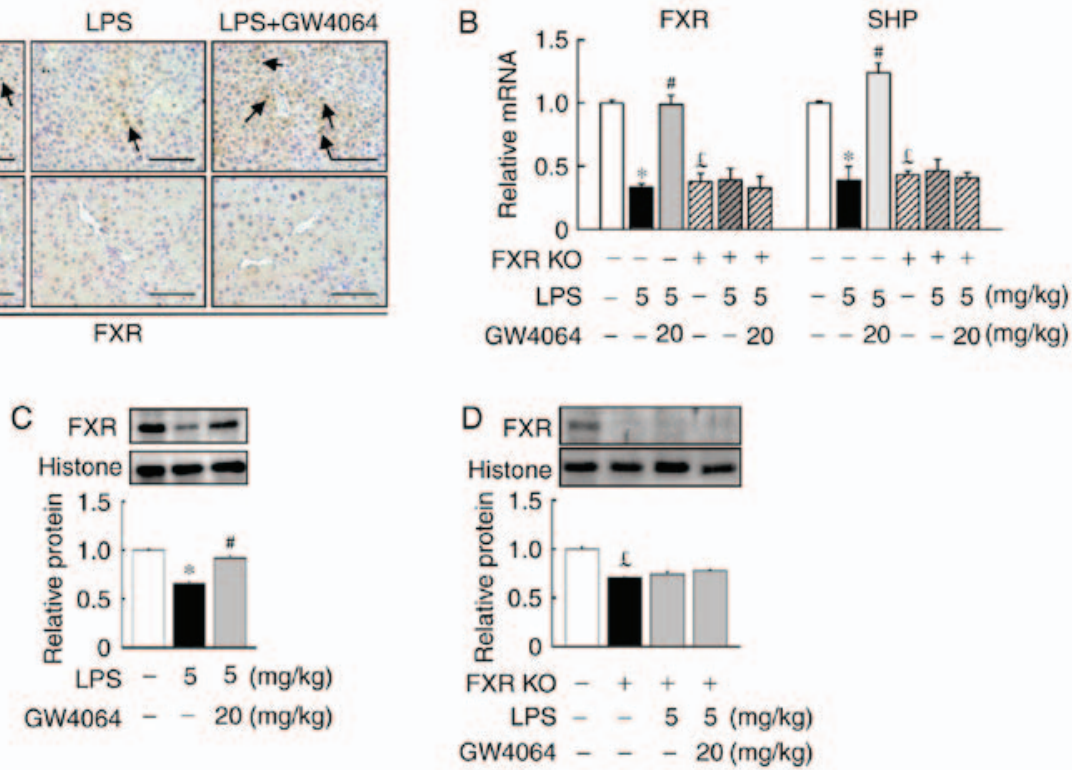

Figure 2. GW4064 administration increases FXR protein levels and mRNA expression in liver tissues of WT mice after LPS injection. WT and FXR KO mice were treated with or without GW4064 $(20 \mathrm{mg} / \mathrm{kg})$ twice after LPS injection $(5 \mathrm{mg} / \mathrm{kg})$. All mice were sacrificed after the last GW4064 injection. (A) Representative immunohistochemical staining images of mouse liver tissues for FXR are displayed. Staining for FXR is indicated by a dark-brown color (black arrows; magnification, x200; scale bar, $100 \mu \mathrm{m}$ ). (B) Hepatic mRNA expression of FXR and SHP were determined by reverse transcription-quantitative polymerase chain reaction analysis. (C and D) FXR proteins were determined by western blot analysis. Histone was used as a control. Expression levels were densitometrically quantified and presented as relative intensity units. Values are expressed as the mean \pm standard error of the mean ( $\mathrm{n}=5 \mathrm{mice}$ for each group). "P<0.05, WT vs. LPS; ${ }^{*} \mathrm{P}<0.05$, LPS vs. LPS + GW4064; ${ }^{\text {} P}<0.05$, WT vs. FXR KO. WT, wild-type; KO, knockout; FXR, farnesoid X receptor; LPS, lipopolysaccharide; SHP, small heterodimer partner. 

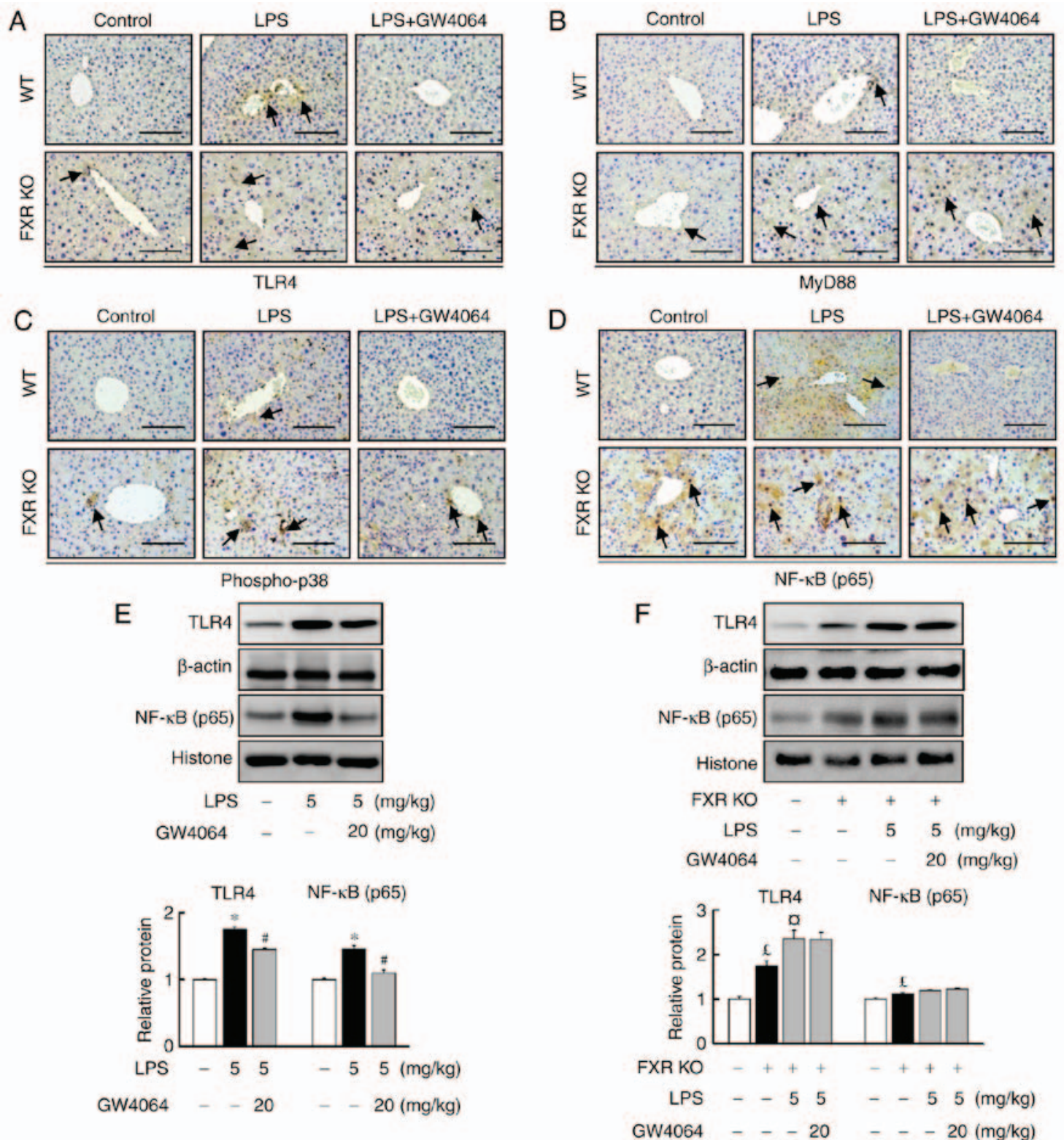

Figure 3. GW4064 attenuates LPS-induced TLR4/MyD88/p38 MAPK/NF-кB pathway in WT mice. WT and FXR KO mice were treated with or without GW4064 (20 mg/kg) twice after LPS injection. At $6 \mathrm{~h}$ following the last GW4064 injection, livers were subjected to IHC staining. Representative images for (A) TLR4, (B) MyD88, (C) p-p38 and (D) NF- $\kappa$ B from mouse liver sections. IHC staining for TLR4, MyD88, p-p38 and NF- $\kappa$ B is indicated by a dark-brown color (indicated by black arrows; magnification, $\mathrm{x} 200$; scale bar, $100 \mu \mathrm{m}$ ). (E and F) TLR4 and NF- $\mathrm{kB}$ protein expression was assessed by western blot analysis . Quantified levels were determined by densitometry and data are presented as relative intensity units. $\beta$-actin and histone were used as controls. Values are expressed as the mean \pm standard error of the mean ( $\mathrm{n}=5$ mice for each group). ${ }^{*} \mathrm{P}<0.05$, WT vs. LPS; ${ }^{\#} \mathrm{P}<0.05$, LPS vs. LPS $+\mathrm{GW} 4064 ;{ }^{\circledR} \mathrm{P}<0.05$, WT vs. FXR $\mathrm{KO} ;{ }^{\circ} \mathrm{P}<0.05$, FXR KO vs. FXR KO + LPS. NF, nuclear factor; p-p38, phosphorylated p38; WT, wild-type; KO, knockout; FXR, farnesoid X receptor; LPS, lipopolysaccharide; MyD88, myeloid differentiation factor 88; TLR, Toll-like receptor; IHC, immunohistochemical.

than in WT mice (Fig. 3). Furthermore, GW4064 treatment did not affect LPS-induced increases in TLR4 and MyD88 protein levels in FXR KO mice (Fig. 3A and B).

LPS injection induced phopho-p38 (Fig. 3C) and NF- $\mathrm{B}$ (Fig. 3D-F) protein levels in WT mice. Treatment with GW4064 markedly inhibited the LPS-induced increases in the protein levels of phopho-p38 and NF- $\mathrm{NB}$ in WT mice (Fig. 3C and D). The induction of hepatic inducible phopho-p38 and NF- $\kappa$ B protein levels by LPS in FXR KO mice was markedly higher than in WT mice. However, treatment with GW4064 did not affect LPS-induced phopho-p38 (Fig. 3C) and NF- $\mathrm{B}$ (Fig. 3D-F) protein levels in FXR KO mice (Fig. $3 \mathrm{C}$ and D). These results indicate that GW4064 reduced the LPS-induced upregulation of the TLR4/p38 MAPK/NF- $\mathrm{B}$ signaling pathway via FXR-dependent signalling in WT mice.

GW4064 inhibits LPS-induced hepatic apoptosis in mice. Stimulation of WT and FXR KO mice with LPS induced similar expression levels of apoptotic proteins and genes. Bax (Fig. 4A) and cytochrome $c$ (Fig. 4B) were significantly higher in FXR KO mice than in WT mice after LPS treatment. In addition, it was confirmed that the activation of apoptotic signaling by LPS was higher in FXR KO mice than in WT mice (Fig. 4A-C). Administration of LPS and FXR deficiency induced the mRNA expression of the major apoptotic gene 

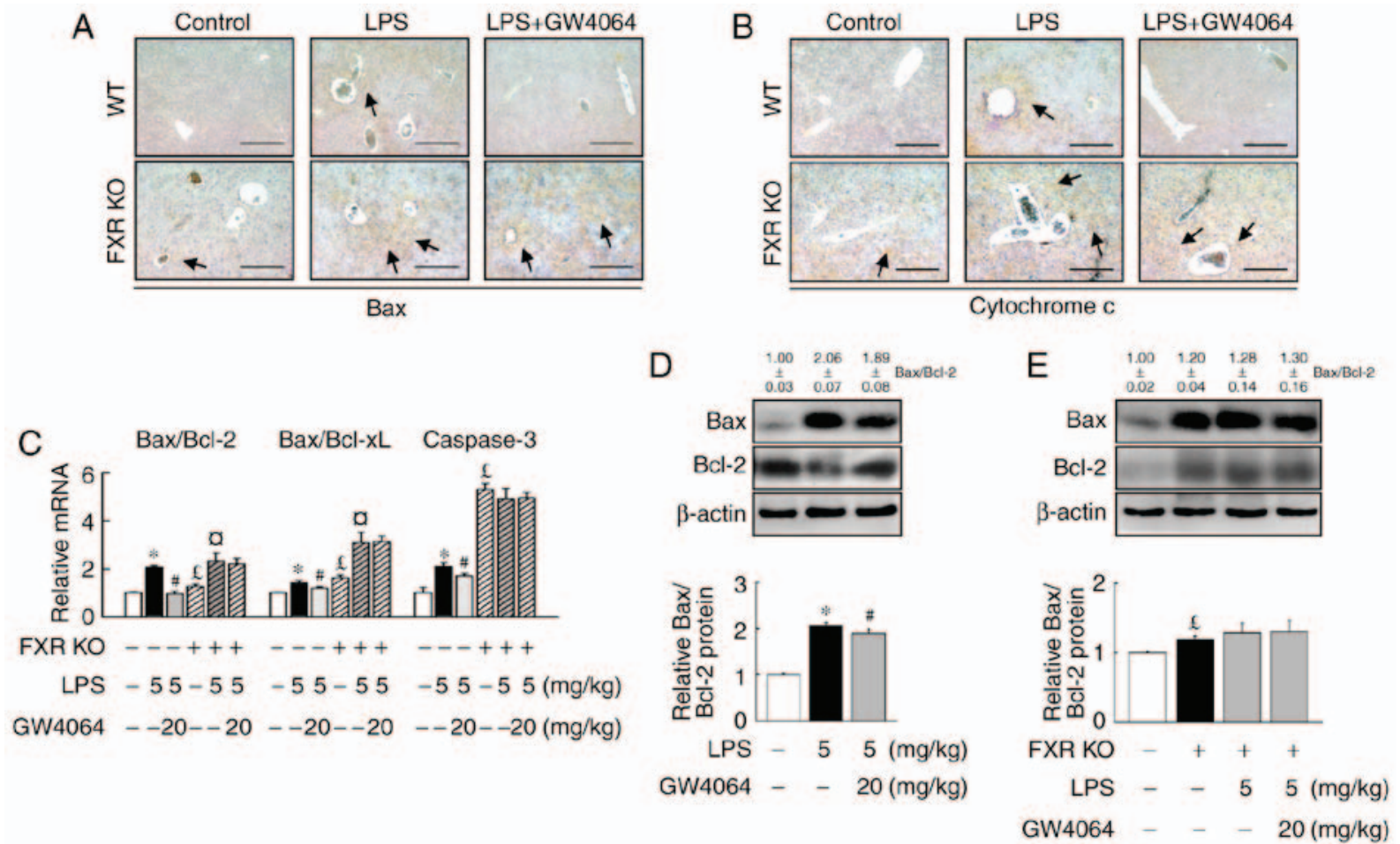

Figure 4. GW4064 attenuates LPS-induced apoptotic factors in WT mice. WT and FXR KO mice were treated with or without GW4064 (20 mg/kg) twice after LPS injection. At $6 \mathrm{~h}$ following the last GW4064 injection, livers were removed for analysis. Representative IHC staining for (A) Bax and (B) cytochrome $c$ from mouse liver sections. IHC staining for Bax and cytochrome $c$ are indicated by a dark-brown color (black arrows; magnification, x100; scale bar, $200 \mu \mathrm{m})$. (C) Total RNA from liver tissues was isolated and the hepatic Bax/Bcl-2 and Bax/Bcl-xL ratio as well as caspase-3 were determined by reverse transcription-quantitative polymerase chain reaction analysis. (D and E) Bax and Bcl-2 protein was determined by western blot analysis. Western blot analyses were scanned by densitometry and data presented as relative intensity units. $\beta$-actin was used as a control. Values are expressed as the mean \pm standard error of the mean ( $\mathrm{n}=5$ mice for each group). ${ }^{\mathrm{P}}<0.05$, WT vs. LPS. ${ }^{\#} \mathrm{P}<0.05$, LPS vs. LPS + GW4064; ${ }^{\mathfrak{f}} \mathrm{P}<0.05$, WT vs. FXR KO; ${ }^{\circ} \mathrm{P}<0.05$, FXR KO vs. FXR KO + LPS Bcl-2, B-cell lymphoma 2; Bax, Bcl-2-associated X protein; Bcl-xL, Bcl extra-large protein; WT, wild-type; KO, knockout; FXR, farnesoid X receptor; LPS, lipopolysaccharide; IHC, immunohistochemical.

caspase-3 (Fig. 4C) and increased the Bax/Bcl-2 and Bax/Bcl extra-large protein (Bcl-xL) mRNA ratios (Fig. 4E). LPS caused a significantly greater increase in the hepatic Bax/Bcl-2 and Bax/Bcl-xL ratio in FXR KO mice compared with that in WT mice (Fig. 4C). GW4064-mediated FXR activation significantly attenuated the LPS-dependent increase of cytochrome $c$ as well as the $\mathrm{Bax} / \mathrm{Bcl}-2$ and $\mathrm{Bax} / \mathrm{Bcl}-\mathrm{xL}$ ratio, and caspase- 3 expression in WT mice, but not in FXR KO mice (Fig. 4). Thus, the results clearly demonstrated that GW4064 has a critical role in suppressing apoptosis associated with LPS-induced liver injury.

\section{Discussion}

Being initially known to regulate liver metabolism, FXR has recently emerged as a factor participating in hepatic inflammation, apoptosis and macrophage infiltration $(12,19)$. However, it has remained to be elucidated whether FXR has a role in LPS-induced hepatic inflammation and apoptosis mediated via the TLR4/MyD88 signaling pathway. The present study applied two strategies, namely the use of the FXR agonist GW4064 and FXR KO mice, and demonstrated that FXR is indispensable for the effect of GW4064 on the TLR4/MyD88 pathway. The present study revealed that GW4064 has an inhibitory effect on TLR4/MyD88-mediated inflammatory factors, including TNF $\alpha$, IL-6, IL-1 $\beta$ and interferon (IFN)- $\gamma$ mRNA expression in WT but not in FXR KO mice. Several lines of evidence suggested that LPS activates NF- $\mathrm{B}$ mobilization and the p38 MAPK pathway through its direct binding to TLR4, ultimately causing the production of pro-inflammatory cytokines such as TNF- $\alpha$ and IL-6 (20,21). NF- $\kappa$ B is activated via MyD88-dependent and -independent pathways, the former of which being more robust and immediate, and the latter accounting for a modest degree in a delayed fashion (22). In addition, the p38 MAPK pathway is also as a key regulator of the expression of inflammatory cytokines, including IL- 6 and TNF- $\alpha$, the main determinant of LPS-stimulated liver damage (23). The results of the present study may be directly associated with a study by Yao et al (12), which demonstrated that FXR agonist GW4064 alleviates endotoxin-induced hepatic inflammation by repressing macrophage activation in mice. The results of the present study illustrate that the FXR counter-regulates the hepatic pro-inflammatory response via the TLR4 signal pathway. It is therefore suggested that FXR represents a unique signaling that differentially regulates the inflammatory response mediated via the TLR4/MyD88 pathway. FXR inhibits the inflammatory response through several mechanisms, including the formation of direct complexes with NF- $\kappa \mathrm{B}$ family members and/or the modulation of MAPK activity $(9,24)$. In addition, GW4064 was reported to alleviate endotoxin-induced hepatic inflammation by repressing macrophage activation (12) and protect against hepatocellular inflammation via induction of suppressor of cytokine signaling 3 (25). Therefore, FXR activation by GW4064 represses the expression of a set of TLR4-regulated genes, including pro-inflammatory cytokines and apoptotic 
factors, and these counter-regulatory effects are lost in FXR $\mathrm{KO}$ mice.

Activation of TLR4 signaling in Kupffer cells may subsequently initiate inflammatory pathways that promote hepatocyte injury, cholestasis, apoptosis and necrosis, as well as activation of stellate cells $(26,27)$. An in vitro study indicated that chenodeoxycholic acid and GW4064, two FXR agonists, rescued HepG2 cells from serum deprivation-induced apoptosis (9). In addition, an in vivo study indicated that FXR activation ameliorated hepatocyte apoptosis during concanavalin A-induced acute liver injury (28). TNF- $\alpha$ and IL-1 $\beta$ induce apoptosis by inducing caspase- 8 and -9 activity, leading to the activation of caspase-3 in this apoptotic cascade (29). The present study provided a functional link between these observations and demonstrated that an increase of apoptosis within the livers of LPS-injected mice is strongly correlated with an increased TLR4 mRNA expression and reduced FXR protein levels. These results, together with the knowledge of the involvement of TLR4 and FXR in hepatic and microphage injury $(9,12,14)$, motivated us to further elucidate the role of TLR4 and FXR in liver cell apoptosis. The present results indicate that LPS treatment led to the upregulation of the pro-apoptotic Bax and downregulation of the anti-apoptotic Bcl-2 in WT mice. It was identified that the pro-inflammatory cytokines TNF- $\alpha$ and IL-1 $\beta$ were elevated alongside LPS-induced liver injury and apoptosis. In addition, treatment with GW4064 alleviated LPS-induced hepatocyte apoptosis in the centrilobular area of the liver of WT mice but not in FXR KO mice.

The present results confirmed that the hepatoprotective action of GW4064 was mediated through FXR activation. Induction of hepatic TNF- $\alpha$ and IFN- $\gamma$ mRNA expression in response to LPS was significantly greater in FXR KO mice compared with that in WT mice, suggesting that certain inflammatory genes are more sensitive to the loss of FXR signaling in mice. Previous studies indicated that FXR KO livers from 9-12 month-old mice displayed prominent liver injury and inflammation (10), and that FXR KO mice are more sensitive to inflammation mediated by $\mathrm{NF}-\kappa \mathrm{B}$ and LPS than WT mice $(9,30)$. Of note, according to another previous study, FXR deficiency completely abolished the ability of FXR agonist WAY-362450 to decrease the elevations of aspartate aminotransferase and ALT. In addition, the protective effect of WAY-362450 on hepatic vascular cell adhesion protein 1, and TIMP metallopeptidase inhibitor $1 \mathrm{mRNA}$ elevation was also lost in the FXR KO mice (31). The present results suggested a potential role of FXR activation in protecting against liver inflammation by modulation of the TLR4 signaling pathway and MyD88 activation in the hepatic inflammatory response. This mutual suppression between FXR and TLR4 signaling may be an important mechanism for preventing liver apoptosis. Therefore, FXR activation may inhibit the increase of TLR4/MyD88 activation through its anti-inflammatory function in the murine liver. Furthermore, it was observed that activation of FXR repressed inflammatory cytokines at the mRNA level, which was associated with changes in the expression of TLR4/MyD88 signaling and NF- $\mathrm{KB}$ activation. Of note, these beneficial effects were seen in WT mice, while they were not detected in FXR KO mice. Taken together, the present results suggested that the protective effects of GW4064 are likely to involve multiple downstream signaling. Regulation of signaling downstream of FXR by GW4064 may provide a novel approach for preventing inflammasome overexpression in order to attenuate hepatic injury. However, to fully dissect the FXR-dependent and -independent functions of GW4064, it is necessary to further assess the function of this compound in FXR KO mice.

\section{Acknowledgements}

This study was supported by grants from the Ministry of Science and Technology, Taipei, Taiwan (grant nos. 102-2320-B182-014, 102-2320-B182-015-MY3 and 103-2320-B182-002-MY3) and the Chang Gung Memorial Hospital (Taoyuan, Taiwan; grant nos. CMRPD1B0262, CMRPD1D0351 and CMRPD1D0352 to T.-Y.L.).

\section{Competing interests}

The authors declare that they have no competing interests.

\section{References}

1. Gao B and Bataller R: Alcoholic liver disease: Pathogenesis and new therapeutic targets. Gastroenterology 141: 1572-1585, 2011.

2. Bryant CE, Spring DR, Gangloff M and Gay NJ: The molecular basis of the host response to lipopolysaccharide. Nat Rev Microbiol 8: 8-14, 2010.

3. Akira S and Takeda K: Toll-like receptor signalling. Nat Rev Immunol 4: 499-511, 2004.

4. Ding N, Zhang Y, Loughran PA, Wang Q, Tsung A and Billiar TR: TIFA upregulation after hypoxia-reoxygenation is TLR4- and MyD88-dependent and associated with HMGB1 upregulation and release. Free Radic Biol Med 63: 361-367, 2013.

5. Wang X, Wang C, Wang J, Zhao S, Zhang K, Wang J, Zhang W, Wu C and Yang J: Pseudoginsenoside-F11 (PF11) exerts anti-neuroinflammatory effects on LPS-activated microglial cells by inhibiting TLR4-mediated TAK1/IKK/NF- $\kappa \mathrm{B}, \mathrm{MAPKs}$ and Akt signaling pathways. Neuropharmacology 79: 642-656, 2014.

6. Wang K: Molecular mechanisms of hepatic apoptosis. Cell Death Dis 5: e996, 2014.

7. Modica S, Gadaleta RM and Moschetta A: Deciphering the nuclear bile acid receptor FXR paradigm. Nucl Recept Signal 8: $\mathrm{e} 005,2010$

8. Calkin AC and Tontonoz P: Transcriptional integration of metabolism by the nuclear sterol-activated receptors LXR and FXR. Nat Rev Mol Cell Biol 13: 213-224, 2012.

9. Wang YD, Chen WD, Wang M, Yu D, Forman BM and Huang W: Farnesoid $\mathrm{X}$ receptor antagonizes nuclear factor kappaB in hepatic inflammatory response. Hepatology 48: 1632-1643, 2008.

10. Yang F, Huang X, Yi T, Yen Y, Moore DD and Huang W: Spontaneous development of liver tumors in the absence of the bile acid receptor farnesoid X receptor. Cancer Res 67: 863-867, 2007.

11. Kim I, Ahn SH, Inagaki T, Choi M, Ito S, Guo GL, Kliewer SA and Gonzalez FJ: Differential regulation of bile acid homeostasis by the farnesoid X receptor in liver and intestine. J Lipid Res 48: 2664-2672, 2007.

12. Yao J, Zhou CS, Ma X, Fu BQ1, Tao LS, Chen M and Xu YP: FXR agonist GW4064 alleviates endotoxin-induced hepatic inflammation by repressing macrophage activation. World $\mathrm{J}$ Gastroenterol 20: 14430-14441, 2014.

13. Wang YD, Yang F, Chen WD, Huang X, Lai L, Forman BM and Huang W: Farnesoid X receptor protects liver cells from apoptosis induced by serum deprivation in vitro and fasting in vivo. Mol Endocrinol 22: 1622-1632, 2008.

14. Ito S, Tanaka Y, Oshino R, Okado S, Hori M and Isobe KI: GADD34 suppresses lipopolysaccharide-induced sepsis and tissue injury through the regulation of macrophage activation. Cell Death Dis 7: e2219, 2016.

15. Xiao HB, Sun ZL, Zhang HB and Zhang DS: Berberine inhibits dyslipidemia in C57BL/6 mice with lipopolysaccharide induced inflammation. Pharmacol Rep 64: 889-895, 2012. 
16. Fiorucci S, Clerici C, Antonelli E, Orlandi S, Goodwin B, Sadeghpour BM, Sabatino G, Russo G, Castellani D, Willson TM, et al: Protective effects of 6-ethyl chenodeoxycholic acid, a farnesoid $\mathrm{X}$ receptor ligand, in estrogen-induced cholestasis. J pharmacol Exp Ther 313: 604-612, 2005.

17. Mencarelli A, Cipriani S, Renga B, D'Amore C, Palladino G, Distrutti E, Baldelli F and Fiorucci S: FXR activation improves myocardial fatty acid metabolism in a rodent model of obesity-driven cardiotoxicity. Nutr Metab Cardiovasc Dis 23 94-101, 2013.

18. Maloney PR, Parks DJ, Haffner CD, Fivush AM, Chandra G, Plunket KD, Creech KL, Moore LB, Wilson JG, Lewis MC, et al: Identification of a chemical tool for the orphan nuclear receptor FXR. J Med Chem 43: 2971-2974, 2000.

19. Zhang DG, Zhang C, Wang JX, Wang BW, Wang H, Zhang ZH, Chen YH, Lu Y, Tao L, Wang JQ, et al: Obeticholic acid protects against carbon tetrachloride-induced acute liver injury and inflammation. Toxicol App Pharmacol 314: 39-47, 2017.

20. Takeuchi $\mathrm{O}$ and Akira S: Pattern recognition receptors and inflammation. Cell 140: 805-820, 2010.

21. McClain CJ, Barve S, Deaciuc I, Kugelmas M and Hill D: Cytokines in alcoholic liver disease. Semin Liver Dis 19: 205-219, 1999.

22. Takeda K and Akira S: TLR signaling pathways. Semin Immunol 16: $3-9,2004$

23. Nagaleekar VK, Sabio G, Aktan I, Chant A, Howe IW, Thornton TM, Benoit PJ, Davis RJ, Rincon M and Boyson JE: Translational control of NKT cell cytokine production by p38 MAPK. J Immunol 186: 4140-4146, 2011.

24. Allen K, Kim ND, Moon JO and Copple BL: Upregulation of early growth response factor- 1 by bile acids requires mitogen-activated protein kinase signaling. Toxicol Appl Pharmacol 243: 63-67, 2010.
25. Xu Z, Huang G, Gong W, Zhou P, Zhao Y, Zhang Y, Zeng Y, Gao M, Pan Z and He F: FXR ligands protect against hepatocellular inflammation via SOCS3 induction. Cell Signal 24: 1658-1664, 2012.

26. Nolan JP: Intestinal endotoxins as mediators of hepatic injury-an idea whose time has come again. Hepatology 10: 887-891, 1989.

27. Zhang P, Zhang M, Wan M, Huang X, Jiang Y, Xu S and Luo M: Tamoxifen attenuates lipopolysaccharide/galactosamine-induced acute liver failure by antagonizing hepatic inflammation and apoptosis. Immunol Invest 46: 284-294, 2017.

28. Lian F, Wang Y, Xiao Y, Wu X, Xu H, Liang L and Yang X: Activated farnesoid $X$ receptor attenuates apoptosis and liver injury in autoimmune hepatitis. Mol Med Rep 12: 5821-5827, 2015.

29. McIlwain DR, Berger T and Mak TW: Caspase functions in cell death and disease. Cold Spring Harb Perspect Biol 5: a008656, 2013.

30. Zollner G, Wagner M, Fickert P, Geier A, Fuchsbichler A, Silbert D, Gumhold J, Zatloukal K, Kaser A, Tilg H, et al: Role of nuclear receptors and hepatocyte-enriched transcription factors for Ntcp repression in biliary obstruction in mouse liver. Am J Physiol Gastrointest Liver Physiol 289: G798-G805, 2005.

31. Zhang S, Wang J, Liu Q and Harnish DC: Farnesoid X receptor agonist WAY-362450 attenuates liver inflammation and fibrosis in murine model of non-alcoholic steatohepatitis. J Hepatol 51: 380-388, 2009.

This work is licensed under a Creative Commons Attribution-NonCommercial-NoDerivatives 4.0 International (CC BY-NC-ND 4.0) License. 